\title{
TEMPERATURE DEPENDENCE OF THE PREEXPONENTIAL FACTORS OF THE FIELD EMISSION FLICKER NOISE CROSS-CORRELATION FUNCTION FOR W(110)K
}

\author{
A.M. DABRoWSKI \\ Institute of Experimental Physics, Wroclaw University \\ 50-205 Wrocław, Cybulskiego 36, Poland
}

AND Ch. KLeinT

Sektion Physik, Leipzig Universität, O-7010 Leipzig, Linnéstrasse 5, Germany

(Received March 19, 1992)

\begin{abstract}
In a field emission tube with two probe-holes the cross-correlation functions of current fluctuations from potassium submonolayers on tungsten have been investigated. The measurements were carried out in the temperature range of $290-430 \mathrm{~K}$ and with coverages between 0.05 and 0.87 . The experimental correlation curves were approximated by a sum of three exponential functions revealing three components with different time constants $\tau_{i}$ and amplitudes $R_{i}$. The temperature dependence of the amplitudes $R_{2}$ and $R_{3}$ is discussed in terms of collective flip-flop processes.
\end{abstract}

PACS numbers: 79.70.+q, 68.90.+g.

\section{Introduction}

The field emission current from adsorbed submonolayers shows fluctuations called the field emission flicker noise (FEFN). They are due to random hopping and surface diffusion processes of the adparticles [1-3]. Using field emission probe-hole techniques with two probe-holes in the fluorescent screen allows the measurement of the collector currents from two different parts of the emitter. Two noise sources are obtained and the correlation between them is investigated [4]. Respective experimental results were presented earlier $[5,6]$. An explanation of the cross-correlation phenomenon was given [5] using an interpretation by adparticle surface diffusion basing on Gomer's assumption of concentration fluctuations caused by surface diffusion in a probed area [2]. It did not explain all experimental facts, foremost not the negative cross-correlation values for certain temperatures 
and coverages. By a fitting procedure the cross-correlation functions (CCFs) are represented by three exponential terms. Their temperature dependence gives new clues to an explanation of the noise in certain ranges of temperature and adparticle coverage.

\section{Cross-correlation functions as a sum of exponential functions}

The cross-correlation (CC) function $R(\tau)$ depends on the temperature and the coverage of potassium in a very strong manner. $R(\tau)$ approaches asymptotically zero with increasing time delay: the faster, the higher the temperature is and the higher is the potassium coverage (Fig. 1). The CC function $R(\tau)$ related to

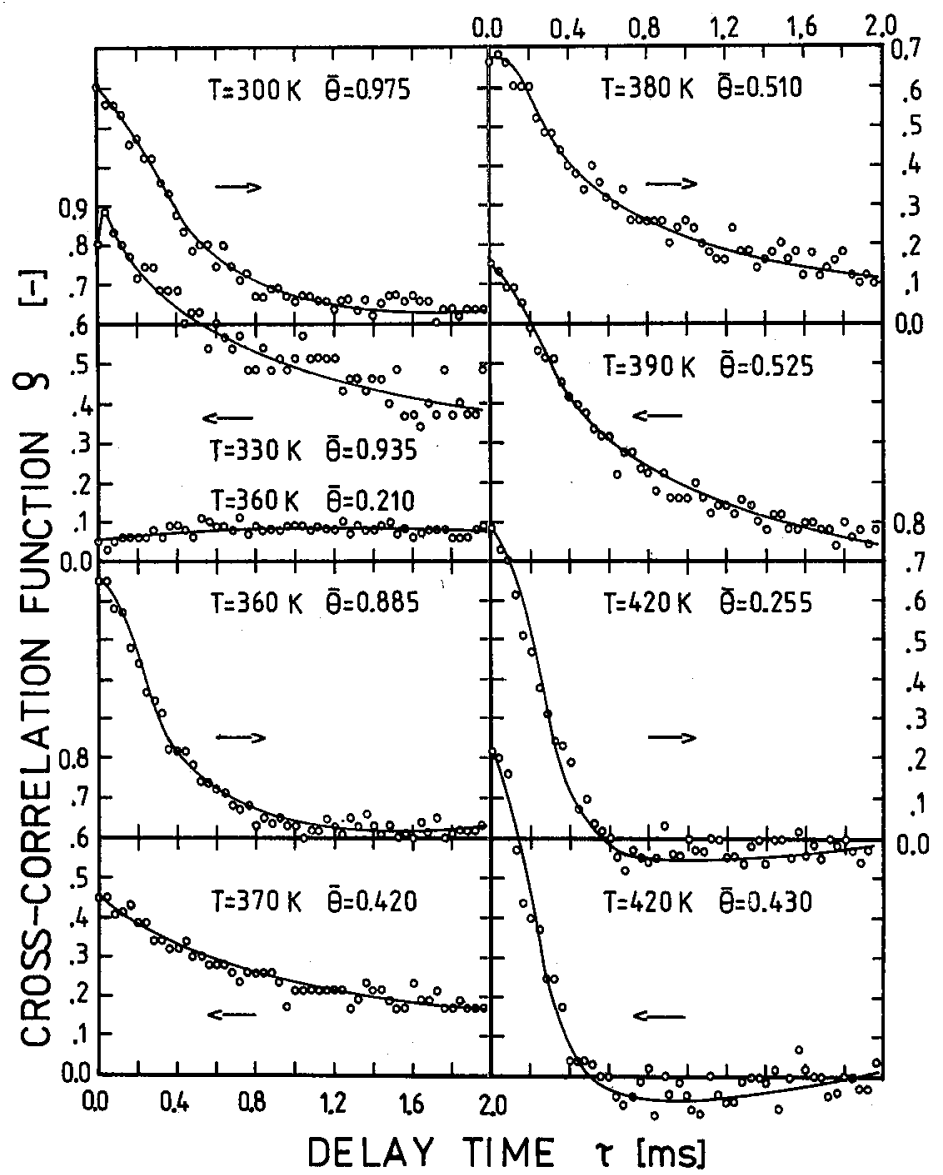

Fig. 1. Experimental normalized cross-correlation functions for a particular set of parameters, indicated in the figure. 
the noise power is called the normalized CC function $\rho(\tau)$ [4]. A distinct correlation maximum is observed at lower coverages. The $\mathrm{CC}$ function assumes negative values at small time delays and low coverages near room temperature.

In a semilogarithmic CCF plot versus time delay we find a part of its points on a straight line. These points can be described by an exponential function which is subtracted from the whole CCF. The outcome of this operation is once more plotted in the semilogarithmic scale and the next exponential function can be fitted. Thus the whole experimental curve is approximated by the sum of three exponential functions

$$
R(\tau)=\sum_{i=1}^{3} R_{i} \exp \left(-\tau_{\mathrm{f}} / \tau_{i}\right),
$$

where we get three components with different decay times $\tau_{i}$ and different amplitudes $R_{i} . R_{1}$ corresponds to the component with the shortest time constant and has negative values. This amplitude is also the origin of negative values of the first CCF points. $R_{2}$ corresponds to medium and $R_{3}$ to long decay components. Both amplitudes are positive.

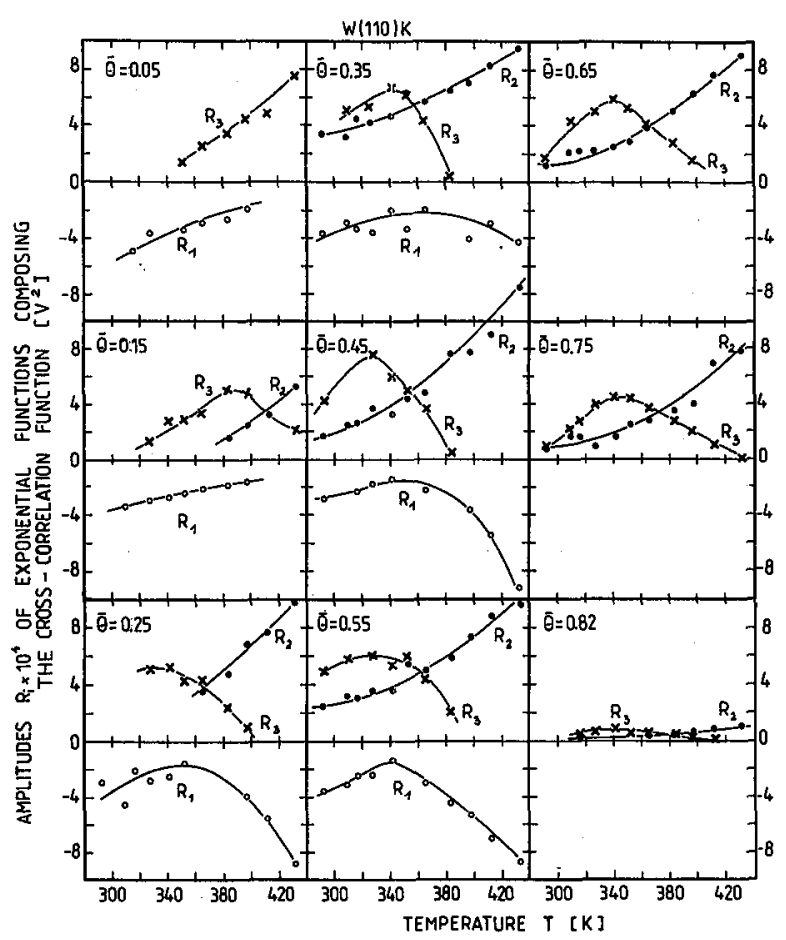

Fig. 2. Temperature dependence of the preexponential factors $R_{i}$ of the exponential functions fitting the experimental cross-correlation functions. 


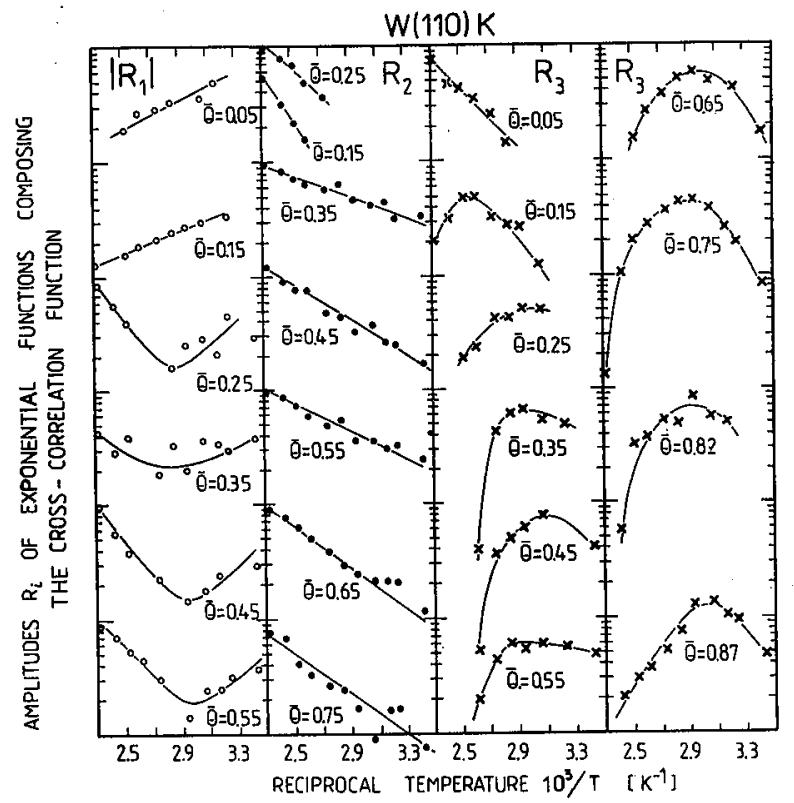

Fig. 3. Semilogarithmic plots of the preexponential factors $R_{i}$ (presented in Fig. 2) versus reciprocal temperature $10^{3} / T$. The vertical scales have been arbitrarily displaced for ease of viewing.

The temperature dependence of the three amplitudes $R_{i}$ is shown in Fig. 2, where the $R_{i}$ are given in square volts and the temperature in Kelvin. The amplitudes $R_{1}$ monotonically rise with temperature to zero only for small coverages for the other coverages they have a maximum. The component $R_{1}$ exists only for coverages up to $\bar{\Theta}=0.6$. This fact expresses the experimental finding that for the higher coverages a CCF maximum is not observed. The second amplitude $R_{2}$ monotonically grows with temperature. $R_{2}$ determines in fact the whole CC curve for the higher temperatures, because the amplitudes $R_{3}$ have a maximum for all coverages. It follows that at room temperature and for small coverages the correlation function is mainly described by the first component $R_{1}$, for high temperatures and coverages, however, it is represented mainly by the second component $R_{2}$.

In Fig. 3 these amplitudes are presented with a semilogarithmic scale versus reciprocal temperature. It demonstrates that all temperature dependences of $R_{2}$ and some for $\left|R_{1}\right|$ and $R_{3}$ are given by straight lines, i.e. they can be described by Arrhenius plots.

\section{Discussion}

The problem that the correlation functions cannot be fitted by the theoretical curves obtained from the surface diffusion theory of field emission fluctuations but can be expressed as the sum of some exponential decays with different time constants, was discussed by Gomer $[2,7]$. 
Let a system have two states $A$ and $B$. Let the mean relaxation times for the states be $\tau_{A}$ and $\tau_{B}$, respectively. A property which influences the cmission should show different values when the system is either in the state $A$ or in the state $B$. The time correlation function for this system will decay exponentially with a relaxation time $\tau_{\mathbf{f}}$ given by [7]

$$
1 / \tau_{\mathrm{f}}=1 / \tau_{A}+1 / \tau_{B}
$$

If a simplified model of these flip-flop processes is used the correlation function is

$$
R(\tau)=c \bar{f}_{A} \bar{f}_{B} g_{1}\left(\tau_{0} / \tau\right) \exp \left(-\tau / \tau_{\mathrm{f}}\right)
$$

where $c$ is a constant, $\bar{f}_{\mathrm{A}}$ and $\bar{f}_{B}$ are the mean probabilities of a molecule being in state $A$ and $B$, respectively. The latter are defined by

$$
\bar{f}_{A}=\tau_{A} /\left(\tau_{A}+\tau_{B}\right) \text { and } \bar{f}_{B}=\tau_{B} /\left(\tau_{A}+\tau_{B}\right),
$$

$g_{1}(\tau)$ is the cross-correlation function obtained by Gomer's adparticle surface diffusion model and $\tau_{0}=r_{0}^{2} / 4 D$, where $r_{0}$ is the radius of the probe region and $D$ is the diffusion coefficient [6]. As it is obvious from Eq. (3) this formula contains both mechanisms of the correlation, the surface diffusion of adparticles and flip-flop processes. Which term of this equation is predominant, depends on the relation between the times $\tau, \tau_{\mathfrak{f}}$ and $\tau_{0}$. If $\tau_{\mathfrak{f}} \ll \tau \approx \tau_{0}$ emphasis lays on diffusion. This means that flip-flop is so rapid relative to diffusion that all flip-flop correlation has been washed out in a time of the order $\tau_{0}$. On the other hand, for $\tau \approx \tau_{\mathrm{f}} \ll \tau_{0}, g_{1}\left(\tau_{0} / \tau\right)$ remains constant so that pure flip-flop dominates the correlation function. For $\tau_{\mathrm{f}} \gg \tau \approx \tau_{0}$ Eq. (3) gives the pure diffusion results. This short discussion illustrates that a coexistence of both mechanisms of the correlation is possible.

Since the experimental CC curve is fitted by the sum of three exponential functions with different time constants a situation seems to prevail where more than two states are involved in the flip-flop processes. However, we have to consider only the second and the third term because the first exponential with $R_{1}$ describes the increase in the correlation function. This latter one depends on the distance between the two collectors [8] and characterizes the time needed for an excitation (or diffusion front in a simple model [9]) to travel between them. Let us assume, therefore, two distinct sets of states, $A \Leftrightarrow B$ and $C \Leftrightarrow D$ interacting pairwise. We now estimate the temperature dependence of the amplitudes $R_{2}$ and $R_{3}$.

$R(0)$ for $\tau=0$ has the form (for one set of states)

$$
R(0)=c \bar{f}_{A} \bar{f}_{B}
$$

which is adequate to the amplitudes $R_{2}$ and $R_{3}$ of the exponential functions fitting the experimental CCFs (see Eq. (3)). The temperature dependence of the relaxation times $\tau_{A}$ and $\tau_{B}$ is assumed to be [10]

$$
1 / \tau_{A}=\nu_{A} \exp \left(-E_{A} / k T\right) \text { and } 1 / \tau_{B}=\nu_{B} \exp \left(-E_{B} / k T\right)
$$

and we have

$$
\bar{f}_{A} \bar{f}_{B}=1 /\left(2+\alpha+\alpha^{-1}\right)
$$

where

$$
\alpha=\tau_{B} / \tau_{A}=\left(\nu_{A} / \nu_{B}\right) \exp \left[\left(E_{B}-E_{A}\right) / k T\right] .
$$


A plot of $\ln \vec{f}_{A} \bar{f}_{B} \equiv \ln R_{2,3}$ versus $1 / \mathrm{T}$ will be linear only if $\alpha \gg 1$ or $\alpha^{-1} \gg 1$ so that

$$
\ln \bar{f}_{A} \bar{f}_{B}=\text { const }+\left|E_{A}-E_{B}\right| / k T .
$$

As obvious from Fig. 3, only the amplitude $R_{2}$ corresponding to the pair of states $A \Leftrightarrow B$ is fully linear for all coverages while $R_{3}$ is linear only for low coverages. This means, the energies of the pair of states $C \Leftrightarrow D$ might change with temperature. Another explanation of this fact can be an influence of the surface diffusion term in Eq. (3) which could show up at higher temperatures as observed [7].

The above explanation of the cross-correlation functions in part by flip-flop processes implies, however, a new and interesting feature. While Chen and Gomer used one collector, our measurements were performed with a two-collector arrangement. Any flip-flop observed by the latter technique (i.e. cross-correlation instead of autocorrelation) seems to need a collective flip-flop to anpear which can be observed with a certain phase shift also in the second collector region. Otherwise, i.e. in case of the earlier investigated single flip-flop processes of independent adparticles [10-12] any many-particle flip-flops would obviously cancel on the average. A. collective or phase controlled flip-flop of many adparticles, however, would strongly support the soliton explanation of certain cross-correlation observations $[9,13,14]$.

\section{Acknowledgement}

The work was supported by the Committee of Researches (KBN), Research Project 4064/PB/IFD/91.

\section{References}

[1] Ch. Kleint, Surf. Sci. 25, 394 and 411 (1971).

[2] R. Gomer, Surf. Sci. 38, 373 (1973).

[3] Ch. Kleint, Surf. Sci. 200, 472 (1988).

[4] J.S. Bendat, Principles and Applications of Random Noise Theory, John Wiley and Sons, New York 1958.

[5] A. Dąbrowski, Ch. Kleint, Surf. Sci. 119, 118 (1982).

[6] A.M. Dąbrowski, Ch. Kleint, Surf. Sci. 172, 372 (1986).

[7] J.R. Chen, R. Gomer, Surf. Sci. 79, 413 (1979).

[8] J. Bęben, Ch. Kleint, R. Męclewski, Surf. Sci. 213, 438 (1989).

[9] Ch. Kleint, Acta Univers. Wratisl. Mat. Fiz. Astron. 936, 81 (1986).

[10] H.-J. Gasse, Ann. Phys. 16, 370 (1965).

[11] Ch. Kleint, K. Mockel, Surf. Sci. 40, 343 (1973).

[12] Ch. Kleint, Phys. Status Solidi A 55, 447 (1979).

[13] J. Bęben, Ch. Kleint, A. Pawełek, Surf. Sci. 213, 451 (1989).

[14] Ch. Kleint, Sci-Tech. Publs. 12, 1 (1990). 